\title{
A Noise Tolerant Zeroing Neural Network for Time-Dependent Complex Matrix Inversion Under Various Kinds of Noises
}

\begin{abstract}
Complex-valued time-dependent matrix inversion (TDMI) is extensively exploited in practical industrial and engineering fields. Many current neural models are presented to find the inverse of a matrix in an ideal noise-free environment. However, the outer interferences are normally believed to be ubiquitous and avoidable in practice. If these neural models are applied to complex-valued TDMI in a noise environment, they need take a lot of precious time to deal with outer noise disturbances in advance. Thus, a noise-suppression model is urgent to be proposed to address this problem. In this study, a complex-valued noise-tolerant zeroing neural network (CVNTZNN) on the basis of an integral-type design formula is established and investigated for finding complex-valued TDMI under a wide variety of noises. Furthermore, both convergence and robustness of the CVNTZNN model are carefully analyzed and rigorously proved. For comparison and verification purposes, the existing zeroing neural network $(\mathrm{ZNN})$ and gradient neural network (GNN) have been presented to address the same problem under the same conditions. Numerical simulation consequences demonstrate the effectiveness and excellence of the proposed CVNTZNN model for complex-valued TDMI under various kinds of noises, by comparing the existing ZNN and GNN models.
\end{abstract}

Index Terms-Zeroing neural network (ZNN), complex-valued matrix inversion, convergence, robustness, time-varying.

\section{INTRODUCTION}

A $\mathrm{S}$ a basic algebraic problem, matrix inversion is widely used in scientific research and practical applications, such as Gaussian regression [1], MIMO [2], [3], robotic arm tracking [4], [5], image processing [6], control engineering [7], [8], etc. In order to solve the matrix inversion accurately, some numerical methods such as Newton iterative method have been proposed and studied [9], [10]. Since the minimum complexity of the numerical iterative algorithms is proportional to the cube of the matrix dimension, they may exist terrible problems such as long running time and too large algorithm complexity, when faced with high-dimensional or large-scale matrix operations.

Unlike numerical methods, contributing to excellent natures including distributed storage, parallel computing, and hardware-implementable features, neural network methods have been extensively developed for various practical applications in the last 30 years. Especially, neural network methods based on potential parallel processing were also successfully applied to solve matrix inversion problems [5], [11]-[16]. In [12], a gradient neural network (GNN) model activated by an odd activation function (AF) is proposed and employed to effectively solve the static matrix inversion. Nevertheless, a certain error occurs while the GNN model [14] is exploited to solve a time-dependent problem. Since the GNN model cannot track the solution at each moment of the time-dependent problem, it can only approach the theoretical inversion infinitely, rather than converging to the exact inversion. To overcome the resultant error of GNN in dynamic situation, a zeroing neural network (ZNN), which can make the most of the time derivative of coefficient matrix to track time-dependent solutions, is proposed and employed to find the accurate solution of time-dependent matrix inversion (TDMI) problem [5], [11], [13]. In addition, the state solution of ZNN, starting from an arbitrary initial state, can globally converge to the theoretical inversion at an exponential rate. In order to speed up the convergence rate, a finite time zeroing neural network model (FTZNN) [17] is carefully constructed and employed for TDMI, and the upper bound of finite convergence time can be calculated theoretically.

Note that GNN, ZNN, and FTZNN models mentioned above were employed to find the inversion of the reversible matrix in an idealized undisturbed environment. However, some external noises inevitably emerge during the hardwareimplementation process of the neural network modes. These noises can be treated as constant noise, random noise, hybrid noise, etc. Although noises can be processed in advance, for example, error compensation can be handled by using a lowpass filter according to the specific time-dependent noise, extra valuable time is wasted. That is to say, considering the noise disturbance in solving the TDMI problem is meaningful for the hardware implementation of the neural network. Thus, some ZNN models with noise-tolerant performance have been researched and developed [18]-[24]. In [18], an integrationenhanced ZNN (IEZNN) model is proposed by Jin et al. and employed to solve the TDMI problem in the real domain, and this IEZNN model can still converge to the theoretical inversion of the TDMI problem when a wide variety of noises are present. Continuing this research, an integrationenhanced noise-tolerant ZNN model has been proposed for solving outer inverse problems in the presence of various kinds of noise, with good performance achieved [25]. In addition, a nonlinearly activated integration design formula has been designed to construct a new ZNN model, which has both finitetime convergence and noise tolerance performance [26], [27]. However, the above mentioned noise-tolerant ZNN models are limited in the real domain.

On the basis of the above researches as well as for better hardware implementation, this paper is devoted to constructing a complex-valued ZNN model to find the inversion of the invertible matrix in the complex domain rather than the real domain. Therefore, a complex-valued noise-tolerant ZNN (CVNTZNN) model is proposed to find the inversion of complex-valued TDMI problem, and its global exponential convergence and noise tolerance are theoretically analyzed. 
Besides, for comparison and verification purposes, GNN and ZNN are also employed to work out the same time-dependent complex-valued matrix inversion problem with the same noise attendance. The comparison results show the superiority of the proposed CVNTZNN model in the existence of a wide variety of noises.

At the end of this part, the primary highlights of this work are exhibited as follows.

1) In this work, a complex-valued noise-tolerant zeroing neural network (CVNTZNN) is designed and analyzed for the complex-valued TDMI solving in the existence of a wide variety of noises.

2) Unlike the traditional GNN and $\mathrm{ZNN}$ models, the proposed CVNTZNN is on the basis of a novel design formula with integral term, which is very different from the design formulas of GNN and ZNN models.

3) The global exponential convergence and robustness of the proposed CVNTZNN model for solving the complexvalued TDMI problem are theoretically proved.

4) It is numerically verified that the proposed CVNTZNN model is still efficient for complex-valued TDMI in the existence of a wide variety of noises, while the above mentioned GNN and ZNN models are no longer valid.

\section{Problem Formulation}

In this paper, we focus on solving the following complexvalued TDMI problem [28]-[33]:

$$
\mathrm{A}(t) \mathrm{K}(t)=I \in \mathbb{C}^{n \times n} \text {, or } \mathrm{K}(t) \mathrm{A}(t)=I \in \mathbb{C}^{n \times n},
$$

where $\mathrm{A}(t) \in \mathbb{C}^{n \times n}$ represents a known time-dependent complex matrix, $\mathrm{K}(t) \in \mathbb{C}^{n \times n}$ represents a target matrix to be figured out, and $I \in \mathbb{C}^{n \times n}$ represents an identity matrix of appropriate size. For the convenience of the rest of this paper, $\mathrm{K}^{-1}(t) \in \mathbb{C}^{n \times n}$ is set to represent the theoretical inversion of (1). It is worth noting that any complex matrix is composed of its real and imaginary parts [34], i.e., $\mathrm{A}(t)=\mathrm{A}_{\mathrm{re}}(t)+i \mathrm{~A}_{\mathrm{im}}(t)$, $\mathrm{K}(t)=\mathrm{K}_{\mathrm{re}}(t)+i \mathrm{~K}_{\mathrm{im}}(t)$ and $I=I_{\mathrm{re}}+i I_{\mathrm{im}}$, where $i=\sqrt{-1}$ represents an imaginary unit. Thus, the complex-valued TDMI (1) can be directly revised as

$$
\left[\mathrm{A}_{\mathrm{re}}(t)+i \mathrm{~A}_{\mathrm{im}}(t)\right]\left[\mathrm{K}_{\mathrm{re}}(t)+i \mathrm{~K}_{\mathrm{im}}(t)\right]=I_{\mathrm{re}}+i I_{\mathrm{im}} .
$$

Considering that the real part and imaginary part on both sides of equation (2) always correspond to each other, we have:

$$
\left\{\begin{array}{l}
\mathrm{A}_{\mathrm{re}}(t) \mathrm{K}_{\mathrm{re}}(t)-\mathrm{A}_{\mathrm{im}}(t) \mathrm{K}_{\mathrm{im}}(t)=I_{\mathrm{re}} \in \mathbb{R}^{n \times n}, \\
\mathrm{~A}_{\mathrm{re}}(t) \mathrm{K}_{\mathrm{im}}(t)+\mathrm{A}_{\mathrm{im}}(t) \mathrm{K}_{\mathrm{re}}(t)=I_{\mathrm{im}} \in \mathbb{R}^{n \times n} .
\end{array}\right.
$$

The compact matrix form of (3) can be revised as

$$
\left[\begin{array}{cc}
\mathrm{A}_{\mathrm{re}}(t) & -\mathrm{A}_{\mathrm{im}}(t) \\
\mathrm{A}_{\mathrm{im}}(t) & \mathrm{A}_{\mathrm{re}}(t)
\end{array}\right]\left[\begin{array}{c}
\mathrm{K}_{\mathrm{re}}(t) \\
\mathrm{K}_{\mathrm{im}}(t)
\end{array}\right]=\left[\begin{array}{c}
I_{\mathrm{re}} \\
I_{\mathrm{im}}
\end{array}\right] \in \mathbb{R}^{2 n \times n} .
$$

Thus, by using the above procedure, the complex-valued TDMI (1) can be transformed into (4) with equal solutions. To make (4) more compact and easy to operate, equation (4) can be rewritten as

$$
\mathrm{P}(t) \chi(t)=M \in \mathbb{R}^{2 n \times n},
$$

where $\mathrm{P}(t) \in \mathbb{R}^{2 n \times 2 n}, \chi(t) \in \mathbb{R}^{2 n \times n}$ and $M \in \mathbb{R}^{2 n \times n}$ are defined as

$\mathrm{P}(t)=\left[\begin{array}{cc}\mathrm{A}_{\mathrm{re}}(t) & -\mathrm{A}_{\mathrm{im}}(t) \\ \mathrm{A}_{\mathrm{im}}(t) & \mathrm{A}_{\mathrm{re}}(t)\end{array}\right], \chi(t)=\left[\begin{array}{c}\mathrm{K}_{\mathrm{re}}(t) \\ \mathrm{K}_{\mathrm{im}}(t)\end{array}\right], M=\left[\begin{array}{c}I_{\mathrm{re}} \\ I_{\mathrm{im}}\end{array}\right]$.

In order to ensure that the theoretical solution $\mathrm{K}^{-1}(t) \in$ $\mathbb{C}^{n \times n}$ of problem (1) is present and unique, we limit the known coefficient matrix $\mathrm{A}(t) \in \mathbb{C}^{n \times n}$ to satisfy $\operatorname{det}(\mathrm{P}(t)) \neq 0$ at each time instant throughout this paper, where the operator $\operatorname{det}(\cdot)$ represents the determinant of $\mathrm{P}(t)$.

\section{GNN AND ZNN MODELS}

For comparison, the traditional gradient neural network (GNN) and zeroing neural network (ZNN) are also constructed for complex-valued TDMI problem (1) solving in this section. Following the conventional GNN's design procedure [28], [31], an energy function $\varepsilon(t)$ is firstly defined as

$$
\varepsilon(t)=\frac{1}{2}\|\mathrm{P}(t) \chi(t)-M\|_{\mathrm{F}}^{2},
$$

where $\|\cdot\|_{F}$ represents the Frobenius norm of a matrix.

By utilizing the negative gradient descent formula $-\frac{\partial \varepsilon(t)}{\partial \chi}$, the traditional GNN model can be obtained [12], [14], [35]:

$$
\dot{\chi}=-\alpha \frac{\partial \varepsilon(t)}{\partial \chi}=-\alpha \mathrm{P}(t)^{\mathrm{T}}(\mathrm{P}(t) \chi(t)-M),
$$

where $(\cdot)^{\mathrm{T}}$ represents the transpose of a matrix, and $\alpha>0$ represents a design parameter.

The design process of the ZNN model is different from the GNN model for solving the complex-valued TDMI problem (1). To monitor and control the solving process of the ZNN model for (1), the matrix-based error function $E(t)$ is firstly defined as below [36]-[40]:

$$
E(t)=\mathrm{P}(t) \chi(t)-M \in \mathbb{R}^{2 n \times n} .
$$

To ensure that each element $e_{i j}(t)$ in $E(t)$ can converge to 0 , the design formula of the $\mathrm{ZNN}$ model is presented as

$$
\frac{\mathrm{d} E(t)}{\mathrm{d} t}=-\alpha E(t) .
$$

Substituting (7) into (8), the traditional ZNN model is attained:

$$
\mathrm{P}(t) \dot{\chi}(t)=-\alpha(\mathrm{P}(t) \chi(t)-M)-\dot{\mathrm{P}}(t) \chi(t),
$$

where the parameter $\alpha$ is defined as before.

When external additive noise is injected, the noise-polluted ZNN model can be written as:

$$
\mathrm{P}(t) \dot{\chi}(t)=-\alpha(\mathrm{P}(t) \chi(t)-M)-\dot{\mathrm{P}}(t) \chi(t)+Z(t),
$$

where $Z(t) \in \mathbb{R}^{2 n \times n}$ represents an external additive noise.

Remark 1: As mentioned in [41], [42], the noise-polluted ZNN mode (10) may not converge to the theoretical inversion of (1). Next, let us theoretically analyze this reason. First, substituting $E(t)=\mathrm{P}(t) \chi(t)-M$ into the noise-polluted ZNN model (10) yields to

$$
\frac{\mathrm{d} E(t)}{\mathrm{d} t}=-\alpha E(t)+Z(t),
$$


of which the $i j$ th subsystem is given as follows:

$$
\frac{\mathrm{d} e_{i j}(t)}{\mathrm{d} t}=-\alpha e_{i j}(t)+z_{i j}(t),
$$

where $e_{i j}(t)$ and $z_{i j}(t)$ represent the $i j$ th element of $E(t)$ and $Z(t)$ respectively. Then, by defining a Lyapnov function candidate $V(t)=\left|e_{i j}(t)\right|^{2} / 2$ for this subsystem, its time derivative can be computed as

$$
\begin{aligned}
\dot{V}(t) & =\frac{\mathrm{d} V(t)}{\mathrm{d} t}=e_{i j}(t) \frac{\mathrm{d} e_{i j}(t)}{\mathrm{d} t} \\
& =e_{i j}(t)\left(-\alpha e_{i j}(t)+z_{i j}(t)\right) \\
& =-\alpha e_{i j}^{2}(t)+e_{i j}(t) z_{i j}(t) \\
& \leq-\alpha e_{i j}^{2}(t)+\left|z_{i j}(t)\right|\left|e_{i j}(t)\right| \\
& =-\alpha\left(\left|e_{i j}(t)\right|-\frac{\left|z_{i j}(t)\right|}{2 \alpha}\right)^{2}+\frac{z_{i j}^{2}(t)}{4 \alpha} .
\end{aligned}
$$

Supposing $\left|e_{i j}(t)\right|>\left|z_{i j}(t)\right| / \alpha$, one can easily obtain

$$
\left|e_{i j}(t)-\frac{\left|z_{i j}(t)\right|}{2 \alpha}\right| \geq\left|e_{i j}(t)\right|-\frac{\left|z_{i j}(t)\right|}{2 \alpha}>\frac{\left|z_{i j}(t)\right|}{2 \alpha},
$$

from which it can be concluded that $\dot{V}(t)<0$. According to this conclusion, equation (12) falls into the following two cases.

1) Once $\left|e_{i j}(t)\right|>\left|z_{i j}(t)\right| / \alpha$ is valid, one could naturally derive that $\dot{V}(t)<0$. Therefore, when $t \rightarrow+\infty$, $e_{i j}(t) \rightarrow 0$.

2) While $\left|e_{i j}(t)\right|>\left|z_{i j}(t)\right| / \alpha$ for some time instant $t$, in view of (12), $\dot{V}(t)$ will be less than a positive value, which means $e_{i j}(t)$ might not converge to 0 as expected. This will lead to two possibilities: $\dot{V}(t) \leq 0$ or $\dot{V}(t)>0$. Even in the worst situation $\dot{V}(t)>0$, $V(t)=\left|e_{i j}\right|^{2} / 2$ will increase, and $\left|e_{i j}(t)\right|$ could not exceed the upper bound $\left|z_{i j}(t)\right| / \alpha$ since $\dot{V}(t)<0$ when $\left|e_{i j}(t)\right|>\left|z_{i j}(t)\right| / \alpha$.

Hence, from the above discussions, it can be readily concluded that $\|E(t)\|_{\mathrm{F}}=\sqrt{\sum_{i=1}^{2 n} \sum_{j=1}^{n} e_{i j}^{2}} \leq \sqrt{2} n\left|z_{i j}(t)\right| / \alpha$.

\section{CVNTZNN MODEL}

In Section III, GNN model (6) and ZNN model (9) were presented to solve the complex-valued TDMI problem (1). In general, GNN model (6) can be used to solve static problems effectively, but while dealing with some time-dependent problems, it may cause a certain lagging error and cannot solve these time-dependent problems accurately. However, ZNN model (9) can solve time-dependent problems accurately in a global exponential manner without noise disturbance. When noise is present, ZNN model (9) may be invalid for solving TDMI problem (1). Hence, a complex-valued noise-tolerant ZNN model (CVNTZNN) with noise-tolerant performance is proposed and analyzed in this section to address this problem.

\section{A. Model design}

In this part, the design process of the CVNTZNN model is listed as follows.

First, an error function $E(t)$ is defined:

$$
E(t)=\mathrm{P}(t) \chi(t)-M \in \mathbb{R}^{2 n \times n} .
$$

Then, unlike the design process of ZNN model (9), a new design formula with an integral term is adopted:

$$
\frac{\mathrm{d} E(t)}{\mathrm{d} t}=-\alpha E(t)-\beta \int_{0}^{t} E(\tau) \mathrm{d} \tau
$$

where $\alpha$ and $\beta$ are adjustable positive parameters.

At last, substituting error function (14) into evolution formula (15), the following CVNTZNN model can be attained:

$$
\begin{aligned}
\mathrm{P}(t) \dot{\chi}(t)= & -\dot{\mathrm{P}}(t) \chi(t)-\alpha(\mathrm{P}(t) \chi(t)-M) \\
& -\beta \int_{0}^{t}(\mathrm{P}(\tau) \chi(\tau)-M) \mathrm{d} \tau .
\end{aligned}
$$

When external additive noises are injected into CVNTZNN model (16), the disturbed CVNTZNN model can be determined by

$$
\begin{aligned}
\mathrm{P}(t) \dot{\chi}(t)= & -\dot{\mathrm{P}}(t) \chi(t)-\alpha(\mathrm{P}(t) \chi(t)-M) \\
& -\beta \int_{0}^{t}(\mathrm{P}(\tau) \chi(\tau)-M) \mathrm{d} \tau+Z(t),
\end{aligned}
$$

where $Z(t) \in \mathbb{R}^{2 n \times n}$ represents external additive noises.

\section{B. Theoretical Analysis without Noises}

In this subsection, the global stability and exponential convergence of the proposed CVNTZNN model (16) will be strictly proved in the absence of noise.

Theorem 1: Given a complex-valued time-dependent invertible matrix $\mathrm{A}(t)$ defined in (1), starting from an arbitrary initial value $\chi(0)$, CVNTZNN model (16) is globally stable in sense of Lyapunov theory.

Proof: Obviously, CVNTZNN model (16) can be simplified as $\dot{E}(t)=-\alpha E(t)-\beta \int_{0}^{t} E(\tau) \mathrm{d} \tau$, which is a compact matrix-form of the following set of $2 n^{2}$ equations:

$$
\dot{e}_{i j}(t)=-\alpha e_{i j}(t)-\beta \int_{0}^{t} e_{i j}(\tau) \mathrm{d} \tau .
$$

Hence, for proving the stability of CVNTZNN model (16), we only need to prove the stability of the $i j$ th subsystem (18). Following [43], the following Lyapunov function candidate can be defined for the $i j$ th subsystem (18):

$$
v_{i j}(t)=e_{i j}^{2}(t)+\beta\left(\int_{0}^{t} e_{i j}(\tau) \mathrm{d} \tau\right)^{2} .
$$

It is obvious that $v_{i j}(t)$ is positive definite; i.e., $v_{i j}(t)>0$ for any $e_{i j}(t) \neq 0$ or $\int_{0}^{t} e_{i j}(\tau) \mathrm{d} \tau \neq 0$, and $v_{i j}(t)=0$ only for $e_{i j}(t)=\int_{0}^{t} e_{i j}(\tau) \mathrm{d} \tau=0$. Thus, its time derivative is computed as

$\frac{\mathrm{d} v_{i j}}{\mathrm{~d} t}=2 e_{i j}(t) \dot{e}_{i j}(t)+2 \beta e_{i j}(t) \int_{0}^{t} e_{i j}(\tau) \mathrm{d} \tau=-2 \alpha e_{i j}^{2}(t) \leq 0$.

Based on the Lyapunov theory, one can conclude that $e_{i j}(t)$ defined in the $i j$ th subsystem (18) globally converges to 0 for any $i \in\{1,2, \cdots, 2 n\}$ and $j \in\{1,2, \cdots, n\}$. Consequently, it can be concluded that $E(t)$ can globally converge to 0 , i.e., the proposed CVNTZNN model (16) can converge to its theoretical solution globally. This proof is complete.

Remark 2: In general, in order to prove the global stability of ZNN, we will define a Lyapunov function. Unlike ZNN, the 
Lyapunov function of the proposed CVNTZNN model for the global stability is defined as $v_{i j}(t)=e_{i j}^{2}(t)+\beta\left(\int_{0}^{t} e_{i j}(\tau) \mathrm{d} \tau\right)^{2}$, and its novelty is to add an integral term $\beta\left(\int_{0}^{t} e_{i j}(\tau) \mathrm{d} \tau\right)^{2}$. The integral term $\beta\left(\int_{0}^{t} e_{i j}(\tau) \mathrm{d} \tau\right)^{2}$ inspired by the design formula ensures the simplicity of its derivative expression, which makes the judgment of negative definiteness very simple.

Theorem 2: Given a complex-valued time-dependent invertible matrix $\mathrm{A}(t)$ defined in (1), the transient solution $\chi(t)$ of CVNTZNN model (16), starting from an arbitrary initial value $\chi(0)$, can exponentially converge to the theoretical solution $A^{-1}(t)$.

Proof: Define $\epsilon(t)=\int_{0}^{t} E(\tau) \mathrm{d} \tau$ and let $e_{i j}(t), \epsilon_{i j}(t)$, $\dot{\epsilon}_{i j}(t), \ddot{\epsilon}_{i j}$ be the $i j$ th element of $E(t), \epsilon(t), \dot{\epsilon}(t), \ddot{\epsilon}(t)$, respectively. Hence, the $i j$ th subsystem of the second-order system $\dot{E}(t)=-\alpha E(t)-\beta \int_{0}^{t} E(\tau) \mathrm{d} \tau$ can be obtained as

$$
\ddot{\epsilon}_{i j}(t)=-\alpha \dot{\epsilon}_{i j}(t)-\beta \epsilon_{i j}(t) \text {. }
$$

Define $\xi_{1}=\left(-\alpha+\sqrt{\alpha^{2}-4 \beta}\right) / 2$ and $\xi_{2}=(-\alpha-$ $\left.\sqrt{\alpha^{2}-4 \beta}\right) / 2$. Seeing that the initial values $\epsilon_{i j}(0)=0$ and $\dot{\epsilon}_{i j}(0)=e_{i j}(0)$, the analytical solution of (19) has the following three cases.

- For $\xi_{1} \neq \xi_{2}$ and $\xi_{1}$ and $\xi_{2}$ being real numbers, i.e., $\alpha^{2}>$ $4 \beta$,

$$
\epsilon_{i j}(t)=\frac{e_{i j}(0)\left(\exp \left(\xi_{1} t\right)-\exp \left(\xi_{2} t\right)\right)}{\sqrt{\alpha^{2}-4 \beta}},
$$

and one further obtains

$$
e_{i j}(t)=\frac{e_{i j}(0)\left(\xi_{1} \exp \left(\xi_{1} t\right)-\xi_{2} \exp \left(\xi_{2} t\right)\right)}{\sqrt{\alpha^{2}-4 \beta}} .
$$

Hence, the error can be regarded as

$$
E(t)=\frac{E(0)\left(\xi_{1} \exp \left(\xi_{1} t\right)-\xi_{2} \exp \left(\xi_{2} t\right)\right)}{\sqrt{\alpha^{2}-4 \beta}} .
$$

- For $\xi_{1}=\xi_{2}$, i.e., $\alpha^{2}=4 \beta$,

$$
\epsilon_{i j}(t)=e_{i j}(0) t \exp \left(\xi_{1} t\right)
$$

and one further obtains

$$
e_{i j}(t)=e_{i j}(0) \exp \left(\xi_{1} t\right)+e_{i j}(0) \xi_{1} t \exp \left(\xi_{1} t\right) .
$$

Hence, the error can be regarded as

$$
E(t)=E(0) \exp \left(\xi_{1} t\right)+E(0) \xi_{1} t \exp \left(\xi_{1} t\right) .
$$

- For $\xi_{1}=\kappa_{1}+i \kappa_{2}$ and $\xi_{2}=\kappa_{1}-i \kappa_{2}$ being conjugate complex numbers, i.e., $\alpha^{2}<4 \beta$,

$$
\epsilon_{i j}(t)=e_{i j}(0) \sin \left(\kappa_{2} t\right) \exp \left(\kappa_{1} t\right) / \kappa_{2},
$$

and one further obtains

$$
e_{i j}(t)=e_{i j}(0) \exp \left(\kappa_{1} t\right)\left(\kappa_{1} \sin \left(\kappa_{2} t\right) / \kappa_{2}+\cos \left(\kappa_{2} t\right)\right) .
$$

Hence, the error can be regarded as

$$
E(t)=E(0) \exp \left(\kappa_{1} t\right)\left(\kappa_{1} \sin \left(\kappa_{2} t\right) / \kappa_{2}+\cos \left(\kappa_{2} t\right)\right) .
$$

Considering the above three cases and Theorem 1 in [44], one can conclude that for the invertible matrix $\mathrm{A}(t)$ defined in (1), the transient solution $\chi(t)$ of CVNTZNN model (17), starting from an arbitrary initial value $\chi(0) \in \mathbb{C}^{n \times n}$, can exponentially converge to the theoretical inverse $A^{-1}(t)$. This proof is complete.

\section{Theoretical Analysis under External Noises}

In this subsection, three theorems are given to study the robustness performance of the disturbed CVNTZNN model (17) when three different kinds of noises are taken into account.

1) Constant Noise: When there is a constant noise, the following theorem can ensure the robustness of the disturbed CVNTZNN model (17).

Theorem 3: Given a complex-valued time-dependent invertible matrix $\mathrm{A}(t)$ defined in (1), when a constant noise is present, the transient solution $\chi(t)$ of the disturbed CVNTZNN model (17), starting from an arbitrary initial value $\chi(0)$, can globally converge to the theoretical solution $A^{-1}(t)$ regardless of the value of constant noise $Z(t)=Z \in \mathbb{R}^{2 n \times n}$.

Proof: For the $i j$ th subsystem of the disturbed CVNTZN$\mathrm{N}$ model (17), when the constant noise $Z$ is present, by using the Laplace transform [45], we have

$$
s e_{i j}(s)-e_{i j}(0)=-\alpha e_{i j}(s)-\frac{\beta}{s} e_{i j}(s)+z_{i j}(s),
$$

i.e.,

$$
e_{i j}(s)=\frac{s\left(e_{i j}(0)+z_{i j}(s)\right)}{s^{2}+s \alpha+\beta} .
$$

Therefore, the transfer function is $s /\left(s^{2}+s \alpha+\beta\right)$ with its poles being $s_{1}=\left(-\alpha+\sqrt{\alpha^{2}-4 \beta}\right) / 2$ and $s_{2}=(-\alpha-$ $\left.\sqrt{\alpha^{2}-4 \beta}\right) / 2$. For $\alpha>0$ and $\beta>0$, we can find that both poles are in the left half-plane, which means that the system is stable. Since constant noise $z_{i j}$ can be viewed as a step signal, its Laplace transform can be solved as $z_{i j}(s)=z_{i j} / s$. For (21), applying the final value theorem [45], we have

$$
\begin{aligned}
\lim _{t \rightarrow \infty} e_{i j}(t) & =\lim _{s \rightarrow 0} s e_{i j}(s) \\
& =\lim _{s \rightarrow 0} \frac{s^{2}\left(e_{i j}(0)+z_{i j} / s\right)}{s^{2}+s \alpha+\beta} \\
& =0 .
\end{aligned}
$$

Thus, one can conclude that $\lim _{t \rightarrow \infty}\|E(t)\|_{\mathrm{F}}=0$. This proof is complete.

2) Linear Noise: In the above, we analyzed the robustness of the disturbed CVNTZNN model (17) under external constant noises. In this part, linear time-dependent noises are considered in the disturbed CVNTZNN model (17). In addition, we have the following theorem to ensure the robustness of the disturbed CVNTZNN model (17) under linear time-dependent noise disturbance.

Theorem 4: Given a complex-valued time-dependent invertible matrix $\mathrm{A}(t)$ defined in (1), when a linear timedependent noise $Z(t)=Z t \in \mathbb{R}^{2 n \times n}$ is present, the transient solution $\chi(t)$ of the disturbed CVNTZNN model (17), starting from an arbitrary initial value $\chi(0) \in \mathbb{C}^{n \times n}$, approaches the theoretical inverse $A^{-1}(t)$ with the upper bound of the steadystate residual error $\lim _{t \rightarrow \infty}\|E(t)\|_{\mathrm{F}}$ being $\|Z\|_{\mathrm{F}} / \beta$. Besides, the error norm $\|E(t)\|_{\mathrm{F}}$ can converge to 0 as $\beta \rightarrow \infty$.

Proof: For the $i j$ th subsystem of the disturbed CVNTZN$\mathrm{N}$ model (17), when the linear time-dependent noise $Z(t)=$ $Z t \in \mathbb{R}^{n \times n}$ is considered, by using the Laplace transform [45], we have

$$
s e_{i j}(s)-e_{i j}(0)=-\alpha e_{i j}(s)-\frac{\beta}{s} e_{i j}(s)+z_{i j} / s^{2},
$$


where $z_{i j} / s^{2}$ is the Laplace transform of $z_{i j} t$. Considering the final value theorem, we further have

$$
\begin{aligned}
\lim _{t \rightarrow \infty} e_{i j}(t) & =\lim _{s \rightarrow 0} s e_{i j}(s) \\
& =\lim _{s \rightarrow 0} \frac{s^{2}\left(e_{i j}(0)+z_{i j} / s^{2}\right)}{s^{2}+s \alpha+\beta} \\
& =\frac{z_{i j}}{\beta} .
\end{aligned}
$$

Thus, one can conclude that

$$
\lim _{t \rightarrow \infty}\|E(t)\|_{\mathrm{F}}=\frac{\|Z\|_{\mathrm{F}}}{\beta} .
$$

Besides, we have $\lim _{t \rightarrow \infty}\|E(t)\|_{\mathrm{F}} \rightarrow 0$ when $\beta \rightarrow \infty$. This proof is complete.

3) Bounded Random Noise: In this part, a bounded random noise is considered in the disturbed CVNTZNN model (17), and we have the following theorem to ensure its robustness.

Theorem 5: Given a complex-valued time-dependent invertible matrix $\mathrm{A}(t)$ defined in (1), when a bounded random noise $Z(t)=\varrho(t) \in \mathbb{R}^{2 n \times n}$ is present, the transient solution $\chi(t)$ of the disturbed CVNTZNN model (17), starting from an arbitrary initial value $\chi(0)$, approaches the theoretical inverse $A^{-1}(t)$ with the steady-state residual error $\lim _{t \rightarrow \infty}\|E(t)\|_{\mathrm{F}}$ bounded by $2 \sqrt{2} n \sup _{0 \leq \tau \leq t}\left|\varrho_{i j}(\tau)\right| / \sqrt{\alpha^{2}-4 \beta}$ for $\alpha^{2}>4 \beta$, or $4 \sqrt{2} n \beta \sup _{0 \leq \tau \leq t}\left|\varrho_{i j}(\tau)\right| /\left(\alpha \sqrt{4 \beta-\alpha^{2}}\right)$ for $\alpha^{2}<4 \beta$, where $\varrho_{i j}(t)$ denotes the $i j$ th element of $\varrho(t)$. In addition, with a suitable value of $\beta, \lim _{t \rightarrow \infty}\|E(t)\|_{\mathrm{F}}$ can be arbitrarily small when $\alpha$ is large enough.

Proof: When a bounded random noise $Z(t)=\varrho(t) \in$ $\mathbb{R}^{2 n \times n}$ is considered, the disturbed CVNTZNN model (17) is transformed as

$$
\dot{E}(t)=-\alpha E(t)-\beta \int_{0}^{t} E(\tau) \mathrm{d} \tau+\varrho(t),
$$

where the $i j$ th subsystem can be written as

$$
\dot{e}_{i j}(t)=-\alpha e_{i j}(t)-\beta \int_{0}^{t} e_{i j}(\tau) \mathrm{d} \tau+\varrho_{i j}(t) .
$$

For different relationships between $\alpha$ and $\beta$, we can consider the following three situations.

- When $\alpha^{2}>4 \beta$, for (22), we have

$$
\begin{aligned}
e_{i j}(t)= & \frac{e_{i j}(0)\left(\xi_{1} \exp \left(\xi_{1} t\right)-\xi_{2} \exp \left(\xi_{2} t\right)\right)}{\left(\xi_{1}-\xi_{2}\right)} \\
& +\left(\int_{0}^{t}\left(\xi_{1} \exp \left(\xi_{1}(t-\tau)\right)-\xi_{2} \exp \left(\xi_{2}(t-\tau)\right)\right)\right. \\
& \left.\varrho_{i j}(\tau) \mathrm{d} \tau\right) \frac{1}{\left(\xi_{1}-\xi_{2}\right)},
\end{aligned}
$$

where $\xi_{1}$ and $\xi_{2}$ are defined as before, i.e., $\xi_{1,2}=$ $\left(-\alpha \pm \sqrt{\alpha^{2}-4 \beta}\right) / 2$. Considering the triangle inequality, we have

$$
\begin{aligned}
\left|e_{i j}(t)\right| \leq & \frac{\left|e_{i j}(0)\left(\xi_{1} \exp \left(\xi_{1} t\right)-\xi_{2} \exp \left(\xi_{2} t\right)\right)\right|}{\left(\xi_{1}-\xi_{2}\right)} \\
& +\frac{\int_{0}^{t}\left|\xi_{1} \exp \left(\xi_{1}(t-\tau)\right)\right| \cdot\left|\varrho_{i j}(\tau)\right| \mathrm{d} \tau}{\left(\xi_{1}-\xi_{2}\right)} \\
& +\frac{\int_{0}^{t}\left|\xi_{2} \exp \left(\xi_{2}(t-\tau)\right)\right| \cdot\left|\varrho_{i j}(\tau)\right| \mathrm{d} \tau}{\left(\xi_{1}-\xi_{2}\right)} .
\end{aligned}
$$

Furthermore, one can obtain

$$
\begin{aligned}
\left|e_{i j}(t)\right| \leq & \frac{\left|e_{i j}(0)\left(\xi_{1} \exp \left(\xi_{1} t\right)-\xi_{2} \exp \left(\xi_{2} t\right)\right)\right|}{\left(\xi_{1}-\xi_{2}\right)} \\
& +\frac{2}{\left(\xi_{1}-\xi_{2}\right)} \max _{0 \leq \tau \leq t}\left|\varrho_{i j}(\tau)\right| \\
= & \frac{\left|e_{i j}(0)\left(\xi_{1} \exp \left(\xi_{1} t\right)-\xi_{2} \exp \left(\xi_{2} t\right)\right)\right|}{\left(\xi_{1}-\xi_{2}\right)} \\
& +\frac{2}{\sqrt{\alpha^{2}-4 \beta}} \max _{0 \leq \tau \leq t}\left|\varrho_{i j}(\tau)\right| .
\end{aligned}
$$

Finally,

$$
\lim _{t \rightarrow \infty}\|E(t)\|_{\mathrm{F}} \leq \frac{2 \sqrt{2} n}{\sqrt{\alpha^{2}-4 \beta}} \sup _{0 \leq \tau \leq t}\left|\varrho_{i j}(\tau)\right| .
$$

- When $\alpha^{2}=4 \beta$, for (22), we have

$$
\begin{aligned}
e_{i j}(t) & =e_{i j}(0) t \xi_{1} \exp \left(\xi_{1} t\right)+e_{i j}(0) \exp \left(\xi_{1} t\right) \\
& +\int_{0}^{t}\left((t-\tau) \xi_{1} \exp \left(\xi_{1}(t-\tau)\right)\right) \varrho_{i j}(\tau) \mathrm{d} \tau \\
& +\int_{0}^{t} \exp \left(\xi_{1}(t-\tau)\right) \varrho_{i j}(\tau) \mathrm{d} \tau,
\end{aligned}
$$

where $\xi_{1}$ is defined as before, i.e., $\xi_{1}=(-\alpha+$ $\left.\sqrt{\alpha^{2}-4 \beta}\right) / 2=-\alpha / 2$. Considering the proof of Theorem 1 in [44], there exist $\mu>0$ and $\nu>0$ such that

$$
\left|\xi_{1}\right| t \exp \left(\xi_{1} t\right) \leq \mu \exp (-\nu t)
$$

On the basis of the inequality (23) and the triangle inequality, one can obtain

$$
\begin{aligned}
\left|e_{i j}(t)\right| \leq & \left|e_{i j}(0)\left(\xi_{1} t \exp \left(\xi_{1} t\right)+\exp \left(\xi_{1} t\right)\right)\right| \\
& +\int_{0}^{t}|\mu \exp (-\nu(t-\tau))| \cdot\left|\varrho_{i j}(\tau)\right| \mathrm{d} \tau \\
& +\int_{0}^{t}\left|\exp \left(\xi_{1}(t-\tau)\right)\right| \cdot\left|\varrho_{i j}(\tau)\right| \mathrm{d} \tau \\
\leq & \left|e_{i j}(0)\left(\xi_{1} t \exp \left(\xi_{1} t\right)+\exp \left(\xi_{1} t\right)\right)\right| \\
& +\left(\frac{\mu}{\nu}-\frac{1}{\xi_{1}}\right) \max _{0 \leq \tau \leq t}\left|\varrho_{i j}(\tau)\right| .
\end{aligned}
$$

Finally,

$$
\lim _{t \rightarrow \infty}\|E(t)\|_{\mathrm{F}} \leq\left(\frac{\mu}{\nu}-\frac{1}{\xi_{1}}\right) \sqrt{2} n \sup _{0 \leq \tau \leq t}\left|\varrho_{i j}(\tau)\right| .
$$

- When $\alpha^{2}<4 \beta$, for (22), we have

$$
\begin{aligned}
e_{i j}(t)= & e_{i j}(0) \exp \left(\kappa_{1} t\right)\left(\kappa_{1} \sin \left(\kappa_{2} t\right) / \kappa_{2}+\cos \left(\kappa_{2} t\right)\right) \\
& +\int_{0}^{t}\left(\kappa_{1} \sin \left(\kappa_{2}(t-\tau)\right) \exp \left(\kappa_{1}(t-\tau)\right) / \kappa_{2}\right. \\
& \left.+\cos \left(\kappa_{2}(t-\tau)\right) \exp \left(\kappa_{1}(t-\tau)\right)\right) \varrho_{i j}(\tau) \mathrm{d} \tau,
\end{aligned}
$$

where $\kappa_{1}$ and $\kappa_{2}$ are defined as $\kappa_{1}=-\alpha / 2$ and $\kappa_{2}=$ $\left.\sqrt{4 \beta-\alpha^{2}}\right) / 2$. On the basis of the triangle inequality, 

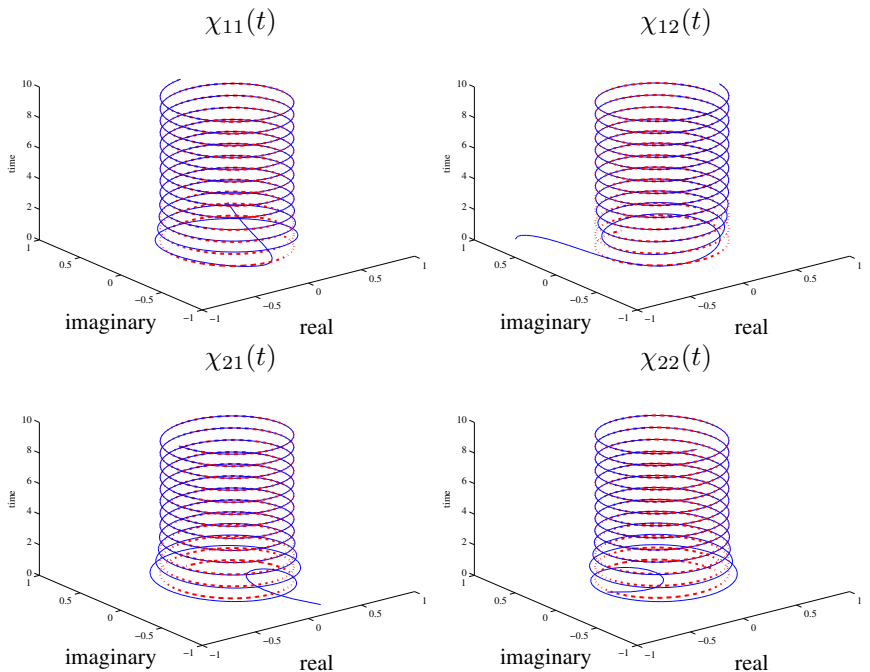

Fig. 1. State output results of the disturbed CVNTZNN model (17) for solving complex-valued TDMI problem (24) with $\alpha=\beta=10$ and noise $Z(t)=$ 0 .

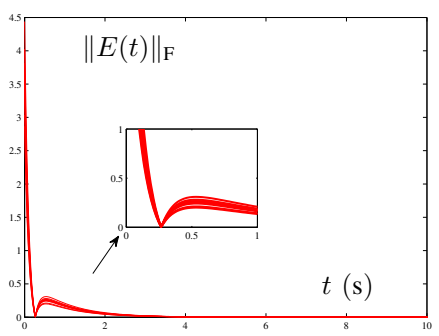

(a) with $\alpha=\beta=10$

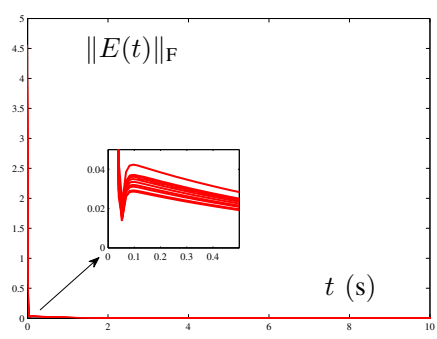

(b) with $\alpha=\beta=100$
Fig. 2. Error norm output results computed by the disturbed CVNTZNN model (17) for solving complex-valued TDMI problem (24) with different design parameters under no noise [i.e., $Z(t)=0$ ].

one can similarly obtain

$$
\begin{aligned}
\left|e_{i j}(t)\right| \leq & \left.\mid e_{i j}(0) \exp \left(\kappa_{1} t\right)\left(\kappa_{1} \sin \left(\kappa_{2} t\right) / \kappa_{2}+\cos \left(\kappa_{2} t\right)\right)\right) \mid \\
& -\frac{\sqrt{\kappa_{1}^{2}+\kappa_{2}^{2}}}{\kappa_{1} \kappa_{2}} \max _{0 \leq \tau \leq t}\left|\varrho_{i j}(\tau)\right| \\
= & \left|e_{i j}(0) \exp \left(\kappa_{1} t\right)\left(\kappa_{1} \sin \left(\kappa_{2} t\right) / \kappa_{2}+\cos \left(\kappa_{2} t\right)\right)\right| \\
& +\frac{4 \beta}{\alpha \sqrt{4 \beta-\alpha^{2}}} \max _{0 \leq \tau \leq t}\left|\varrho_{i j}(\tau)\right| .
\end{aligned}
$$

Finally,

$$
\lim _{t \rightarrow \infty}\|E(t)\|_{\mathrm{F}} \leq \frac{4 \sqrt{2} \beta n}{\alpha \sqrt{4 \beta-\alpha^{2}}} \sup _{0 \leq \tau \leq t}\left|\varrho_{i j}(\tau)\right| .
$$

From the above discussion, one can conclude that the error norm $\|E(t)\|_{\mathrm{F}}$ of the disturbed CVNTZNN model (17) is bounded when the the bounded random noises $\varrho(t)$ is injected. Besides, the steady-state error norm $\lim _{t \rightarrow \infty}\|E(t)\|_{\mathrm{F}}$ of the disturbed CVNTZNN model (17) is bounded by $2 \sqrt{2} n \sup _{0 \leq \tau \leq t}\left|\varrho_{i j}(\tau)\right| / \sqrt{\alpha^{2}-4 \beta}$ for $\alpha^{2}>4 \beta$, or $4 \sqrt{2} n \beta \sup _{0 \leq \tau \leq t}\left|\varrho_{i j}(\tau)\right| /\left(\alpha \sqrt{4 \beta-\alpha^{2}}\right)$ for $\alpha^{2}<4 \beta$. In other words, with a suitable size of $\beta, \lim _{t \rightarrow \infty}\|E(t)\|_{\mathrm{F}}$ can be arbitrarily small, when when $\alpha$ is large enough. This proof is complete.
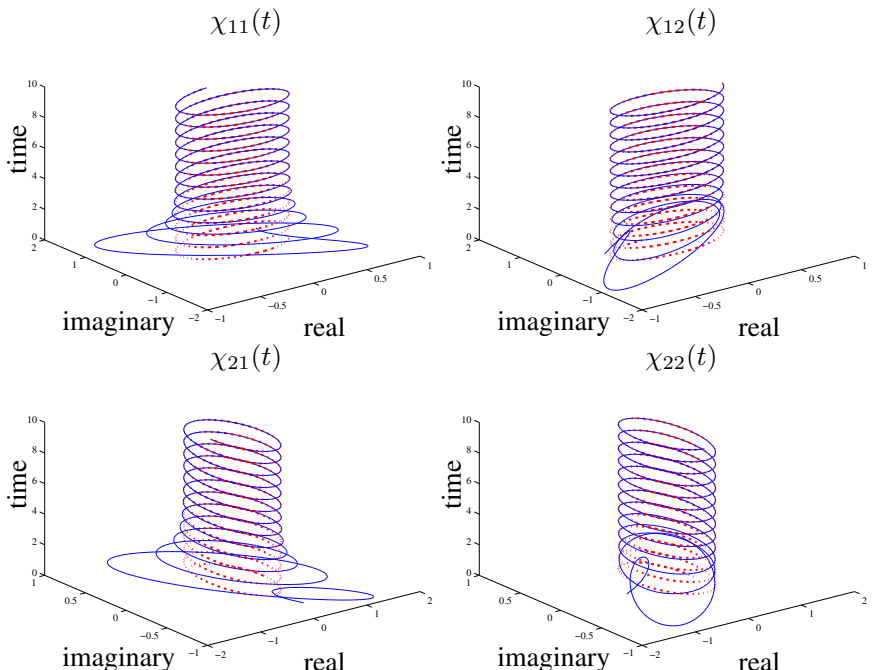

Fig. 3. State output results of the disturbed CVNTZNN model (17) for solving complex-valued TDMI problem (24) with $\alpha=\beta=10$ and constant noise $z_{i j}(t)=10$.

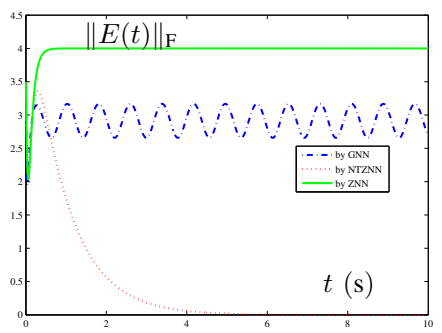

(a) with $\alpha=\beta=10$

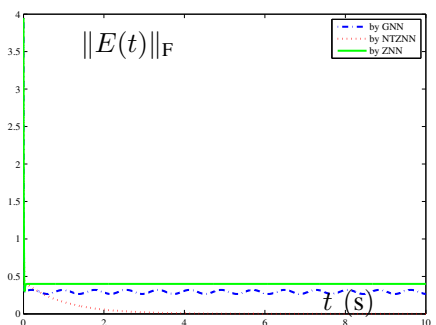

(b) with $\alpha=\beta=100$
Fig. 4. Error norm output results computed by the disturbed CVNTZNN model (17) GNN model (6) and ZNN model (9) for solving complex-valued TDMI problem (24) with different design parameters under constant noise $z_{i j}(t)=10$.

TABLE I

CATEGORIES OF NOISE USED IN THE PAPER

\begin{tabular}{ccccc}
\hline Type & Constant & Linear & Random & Time-varying \\
\hline Example 1 & 10 & $0.8 t$ & {$[14,16]$} & $0.5 \sin (0.1 t)$ \\
\hline Example 2 & 10 & $t$ & {$[-0.5,0.5]$} & $0.1 \exp (0.1 t)$ \\
\hline
\end{tabular}

\section{Comparative Verification}

To solve complex-valued TDMI problem (1), in Sections III and IV, GNN model (6), ZNN model (9), and CVNTZNN model (17) were presented. For comparison and illustration purposes, in this section, two numerical examples are used to verify the validity and superiority of the disturbed CVNTZNN model (17) when various noises are injected. Besides, the category of noises is shown in Tab. I.

\section{A. Example I}

First of all, the following complex time-dependent matrix is considered [40]:

$$
\mathrm{A}(t)=\left[\begin{array}{cc}
\exp (8 i t) & -i \exp (-8 i t) \\
-i \exp (8 i t) & \exp (-8 i t)
\end{array}\right] .
$$

In order to verify the validity of the disturbed CVNTZNN model (17), the corresponding theoretical inverse $\mathrm{A}^{-1}(t)$ of 


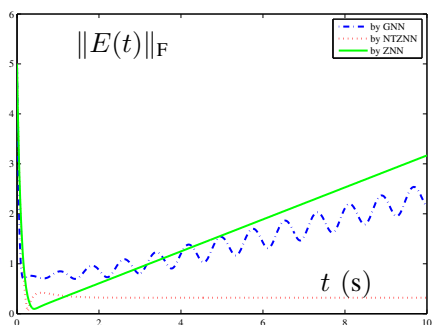

(a) with $\alpha=\beta=10$

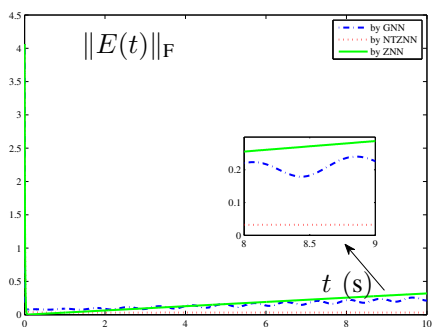

(b) with $\alpha=\beta=100$

Fig. 5. Error norm output results computed by the disturbed CVNTZNN model (17) GNN model (6) and ZNN model (9) for solving complex-valued TDMI problem (24) with different design parameters under linear noise $z_{i j}(t)=0.8 t$.

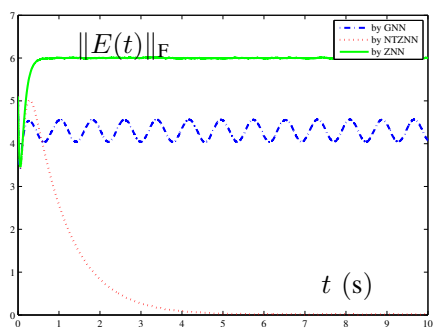

(a) with $\alpha=\beta=10$

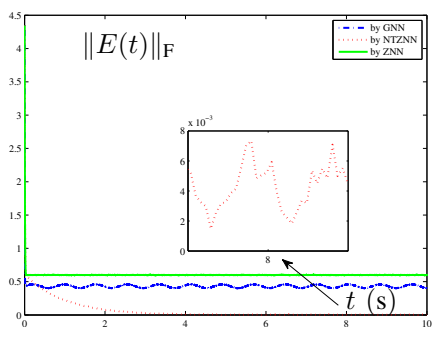

(b) with $\alpha=\beta=100$

Fig. 6. Error norm output results computed by the disturbed CVNTZNN model (17) GNN model (6) and ZNN model (9) for solving complex-valued TDMI problem (24) with different design parameters under random noise $z_{i j}(t)=[14,16]^{2 \times 2}$.

the above complex-valued matrix can be calculated as

$$
\mathrm{A}^{-1}(t)=\left[\begin{array}{cc}
0.5 \exp (8 i t) & 0.5 i \exp (8 i t) \\
0.5 i \exp (-8 i t) & 0.5 \exp (-8 i t)
\end{array}\right]
$$

Besides, according to the specific expression of $\mathrm{A}(t)$, we can obtain the corresponding $\mathrm{P}(t)$ as

$$
\mathrm{P}(t)=\left[\begin{array}{cccc}
\cos (8 t) & -\sin (8 t) & -\sin (8 t) & \cos (8 t) \\
\sin (8 t) & \cos (8 t) & \cos (8 t) & \sin (8 t) \\
\sin (8 t) & -\cos (8 t) & \cos (8 t) & -\sin (8 t) \\
-\cos (8 t) & -\sin (8 t) & \sin (8 t) & \cos (8 t)
\end{array}\right] .
$$

We first check the effectiveness and convergence of the disturbed CVNTZNN model (17) for solving this example without noise [i.e., $Z(t)=0$ ]. With $\beta=\alpha=10$, the corresponding simulation outputs are demonstrated in Fig. 12. The solid blue line in Fig.1 represents state solution of the disturbed CVNTZNN model (17), while the red dotted line represents the theoretical inverse $\mathrm{A}^{-1}(t)$ of (24). As observed from Fig. 1, the solid blue lines in all subgraphs can quickly coincide with the solid red line in about $6 \mathrm{~s}$. That is, the state solution $\chi(t)$ starting from an arbitrary initial matrix $\chi(0) \in[-1,1]^{2 \times 2}$ can converge to the theoretical solution $\chi^{-1}(t)$ accurately. To fully verify the excellent convergence of the disturbed CVNTZNN model (17), the transient state of the error norm $\|E(t)\|_{\mathrm{F}}$ is demonstrated in Fig. 2. As observed from Fig. 2(a), all of error norms $\|E(t)\|_{\mathrm{F}}$ of the disturbed CVNTZNN model (17) with 10 arbitrary initial states $\chi(0) \in[-1,1]^{2 \times 2}$ for $(24)$ can descend to 0 within 6 s. Increasing the design parameters from $\beta=\alpha=10$ to $\beta=\alpha=100$, we can find from Fig. 2(b) that all

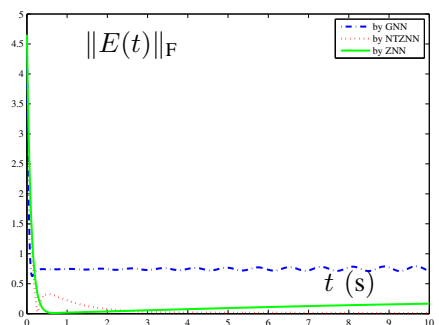

(a) with $\alpha=\beta=10$

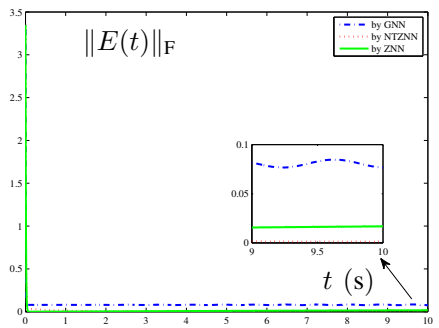

(b) with $\alpha=\beta=100$
Fig. 7. Error norm output results computed by the disturbed CVNTZNN model (17) GNN model (6) and ZNN model (9) for solving complex-valued TDMI problem (24) with different design parameters under time-varying noise $z_{i j}(t)=0.5 \sin (0.1 t)$.

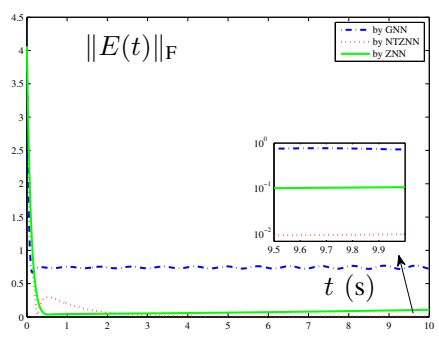

(a) with $\alpha=\beta=10$

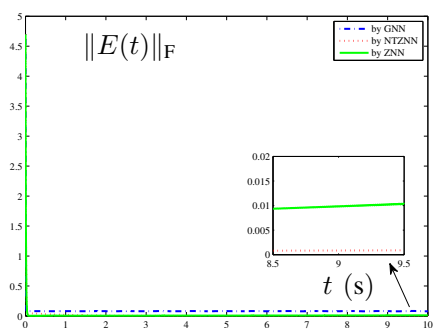

(b) with $\alpha=\beta=100$
Fig. 8. Error norm output results computed by the disturbed CVNTZNN model (17) GNN model (6) and ZNN model (9) for solving complex-valued TDMI problem (24) with different design parameters under time-varying noise $z_{i j}(t)=0.1 \exp (0.1 t)$.

of the 10 residual errors can descend rapidly to 0 . These simulation results are consistent with the above mentioned global exponential convergence.

In practical applications, noise disturbances are ubiquitous and unavoidable. For comparison and verification purposes, several types of noises are also considered in this simulation part. GNN model (6) and ZNN model (9) are also employed to solve (24) under the same noise interferences, and the simulation results are demonstrated in Fig. 3-8.

When a constant noise $z_{i j}(t)=10$ is injected, with $\alpha=\beta=10$ and starting from an arbitrary initial matrix $\chi(0)$, the transient path of the state solution $\chi(t)$ for the disturbed CVNTZNN model (17) is demonstrated in Fig. 3, from which it can be concluded that the solid blue line [i.e., state solution $\chi(t)]$ coincides well with the red dashed line [i.e., theoretical solution $\left.\chi^{-1}(t)\right]$ after about $6 \mathrm{~s}$. In other words, the state solution $\chi(t)$ of the disturbed CVNTZNN model (17) can converge to the theoretical solution $\chi^{-1}(t)$ when polluted by a constant noise $z_{i j}(t)=10$. Fig. 4 shows error norm output results computed by the disturbed CVNTZNN model (17) GNN model (6) and ZNN model (9) for solving complexvalued TDMI problem when polluted by the constant noise $z_{i j}(t)=10$. As demonstrated in Fig. 4(a), the error norm of the disturbed CVNTZNN model (17) drops to 0 within about $6 \mathrm{~s}$ even if it is disturbed by noise $z_{i j}(t)=10$. On the contrary, the error norms of ZNN model (9) and GNN model (6) cannot converge to 0 . There is a large error for these two models. Increasing the values of $\beta$ and $\alpha$ from 10 to 100 (i.e., $\alpha=\beta=100$ ), as demonstrated in Fig. 4(b), the convergence speed of the error norm for the disturbed CVNTZNN model 


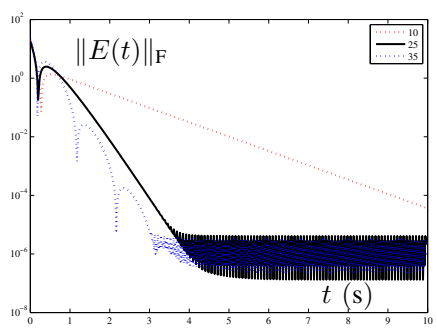

(a) by noise $z(t)=0$.

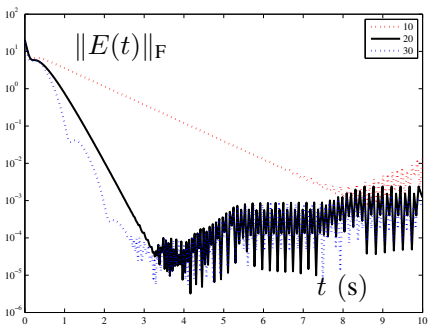

(b) by constant noise $z(t)=10$.

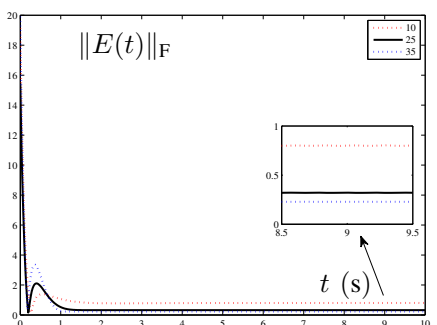

(c) by linear noise $z(t)=t$.

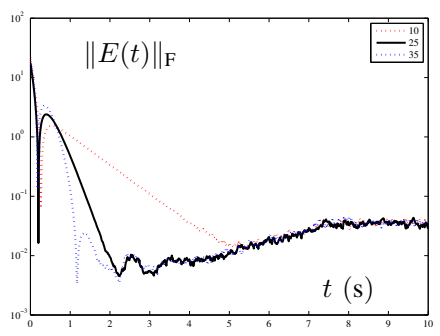

(d) by random noise $-0.5 \leq z(t) \leq$ 0.5

Fig. 9. Error norm output results computed by the disturbed CVNTZNN model (17) for solving complex-valued TDMI problem (24) with the same $\alpha$ $[i . e ., \alpha=10]$ and different $\beta[$ i.e., $\beta=10,25,35]$ under different noises.

(17) is further accelerated, while the error norms of GNN model (6) and ZNN model (9) still cannot converge to 0.

When a linear time-dependent noise $z_{i j}(t)=0.8 t$ is injected, the error norms $\|E(t)\|_{\mathrm{F}}$ of the disturbed CVNTZNN model (17), GNN model (6), and ZNN model (9) for solving (24) are demonstrated in Fig. 5. As illustrated in Fig.5(a), with $\alpha=\beta=10$, the error norm convergence of the disturbed CVNTZNN model (17) is best among these three neural models. In addition, increasing the values of $\beta$ and $\alpha$ from 10 to 100 (i.e., $\alpha=\beta=100$ ), simulation results plotted in Fig. 5(b) further validate the dominance of the disturbed CVNTZNN model (17) under noise $z_{i j}(t)=0.8 t$, as compared with GNN model (6) and ZNN model (9).

When a bounded random noise $z_{i j}(t) \in[14,16]$ is injected, with $\alpha=\beta=10$, the error norms $\|E(t)\|_{\mathrm{F}}$ computed by the disturbed CVNTZNN model (17), GNN model (6), and ZNN model (9) for solving (24) are illustrated in Fig. 6. As illustrated in Fig. 6(a), the error norm $\|E(t)\|_{\mathrm{F}}$ of the disturbed CVNTZNN model (17) rapidly drops to 0 , and the convergence time is about $6 \mathrm{~s}$. On the contrary, the error norm $\|E(t)\|_{\mathrm{F}}$ of $\mathrm{ZNN}$ (9) can only drop to about 6 , and the error norm of GNN model (6) drops to about 4.2 and keeps fluctuating. Increasing the values of $\beta$ and $\alpha$ from 10 to 100 (i.e., $\alpha=\beta=100$ ), as illustrated in Fig. 6(b), the error norm of the disturbed CVNTZNN model (17) rapidly drops to 0 at a faster rate with the order of $10^{-3}$, whereas error norms of ZNN model (9) and GNN model (6) only drops to around 0.5.

In addition, we further consider two different types of timedependent noises [i.e., $z_{i j}(t)=0.5 \sin (0.1 t)$ and $z_{i j}(t)=$ $0.1 \exp (0.1 t)]$, and the corresponding simulation results are illustrated in Figs. 7 and 8. Similar to the previous mentioned situations, Figs. 7 and 8 further validate the superiority of the disturbed CVNTZNN model (17) to ZNN model (9) and GNN model (6) under these two types of time-dependent noises.

In summary, the comparison results authenticate the superiority of the proposed CVNTZNN model (17) for solving (24) compared to ZNN model (9) and GNN model (6), regardless of the disturbed noise is constant noise, linear time-dependent noise, bounded random noise, or nonlinear time-dependent noise or unbounded time-dependent noise.

\section{B. Example II}

To further verify the excellent robustness of the disturbed CVNTZNN model (17), a higher dimensional Toeplitz matrix
$\mathrm{A}(t) \in \mathbb{C}^{4 \times 4}$ is considered as following:

$$
\mathrm{A}(t)=\left[\begin{array}{cccc}
4+\sin (i t) & \cos (i t) & \cos (i t) / 2 & \cos (i t) / 3 \\
\cos (i t) & 4+\sin (i t) & \cos (i t) & \cos (i t) / 2 \\
\cos (i t) / 2 & \cos (i t) & 4+\sin (i t) & \cos (i t) \\
\cos (i t) / 3 & \cos (i t) / 2 & \cos (i t) & 4+\sin (i t)
\end{array}\right]
$$

The corresponding matrix $\mathrm{P}(t)$ from (26) contains 64 elements and is omitted here. In this example, the design parameter $\alpha$ is set to $\alpha=10$, and $\beta$ is set to $\beta=10,25,35$, which represent three cases of $\alpha^{2}-4 \beta>0, \alpha^{2}-4 \beta=0$ and $\alpha^{2}-4 \beta<0$, respectively.

The simulation results computed by the disturbed CVNTZNN model (17) for solving the complex-valued TDMI problem (26) are demonstrated in Fig. 9. Considering that when there is noise disturbance, ZNN model (9) and GNN model (6) have a large error in solving the TDMI problem, so their simulations are omitted in this example. As demonstrated in Fig. 9(a), error norms $\|E(t)\|_{\mathrm{F}}$ of the disturbed CVNTZNN model (17) for solving complex matrix (26) can converge to 0 accurately (the order is $10^{-6}$ ) when there is no noise disturbance. Besides, as demonstrated in Fig. 9(b), when polluted by a constant noise, the error norms $\|E(t)\|_{\mathrm{F}}$ of the disturbed CVNTZNN model (17) for solving complex matrix (26) can approach to 0 quickly. Also, as demonstrated in Fig. 9(c), when disturbed by a linear noise, error norms of the disturbed CVNTZNN model (17) can approach 0 quickly and remain stable (no rising trend). In addition, the larger the parameter $\beta$ is, the smaller the error norm is. Moreover, as demonstrated in Fig. 9(d), when disturbed by a random noise, error norms of the disturbed CVNTZNN model (17) for solving the complex-valued TDMI problem (26) can converge to 0 in an accurate way (order is $10^{-2}$ ). In summary, the comparison results of this example verify the validity and accuracy of the disturbed CVNTZNN model (17) for solving the complex-valued TDMI problem (26) under various noise perturbations.

\section{Conclusion}

To find the accurate inverse of complex-valued timedependent matrix in the complex domain, a complex-valued noise-tolerant zeroing neural network (CVNTZNN) is designed and proposed in this paper. The detailed theoretical analyses of the CVNTZNN model are discussed when different types noises are considered. It is proved that the CVNTZN$\mathrm{N}$ model is robust and convergent to the theoretical solution 
accurately of the complex-valued time-dependent matrix under various noises attendance. Furthermore, for comparison as well as verification, the GNN and ZNN models are also employed to deal with the same time-dependent complex-valued matrix inversion problem under the same noise disturbance. The comparison of the two examples verify the validity and excellence of the proposed CVNTZNN model. The future directions of this work may include the convergence speedup by adding nonlinear activation functions, model discretization, and hardware implementations.

\section{REFERENCES}

[1] Y. Zhang and W.E. Leithead, "Exploiting Hessian matrix and trust-region algorithm in hyperparameters estimation of Gaussian process," Appl. Math. Comput., vol. 171, no. 2, pp. 1264-1281, 2005.

[2] M. Wu, B. Yin, A. Vosoughi, and C. Studer, "Approximate matrix inversion for high-throughput data detection in the large-scale MIMO uplink," Proceedings of IEEE International Symposium on Circuits and Systems., pp. 2155-2158, 2013.

[3] L. Ma, K. Dickson, J. Mcallister, and J. Mccanny, "QR decompositionbased matrix inversion for high performance embedded MIMO receivers," IEEE Trans. Signal Process., vol. 59, no. 4, pp. 1858-1867, 2011.

[4] W. Fang, Y. Zhen, B. Kang, S. Xi, and L. Shang, "A simulation research on the visual servo based on Pseudo-Inverse of image Jacobian matrix for robot," Appl. Mech. Mater., vol. 494-495, pp.1212-1215, 2014.

[5] D. Guo and Y. Zhang, "Zhang neural network, Getz-Marsden dynamic system, and discrete-time algorithms for time-varying matrix inversion with application to robots," Neurocomputing, vol. 97, pp. 22-32, 2012.

[6] I. Dobbins and T. James, "Matrix inversion tomosynthesis improvements in longitudinal X-ray slice imaging," U.S., 1990.

[7] F. Ding, and H. Zhang, "Gradient-based iterative algorithm for a class of the coupled matrix equations related to control systems," IET Contr. Theor. Appl., vol. 8, no. 15, pp. 1588-1595, 2014.

[8] L. Liu, Y. Liu, S. Tong, "Neural networks-based adaptive finite-time faulttolerant control for a class of strict-feedback switched nonlinear systems," IEEE Trans. Cybern., vol. 49, no. 7, pp. 2536-2545, 2019.

[9] H. Rajbenbach, Y. Fainman, and S.H. Lee, "Optical implementation of an iterative algorithm formatrix inversion," Appl. Opt., vol. 26, no. 6, pp. 1024-1031, 1987.

[10] E.G. Forsythe and R.A. Leibler, "Matrix inversion by a Monte Carlo method," Mathematical Tables and Other Aids to Computation, vol. 4, no. 31, pp. 127-129, 1950.

[11] Y. Zhang and S.S. Ge, "Design and analysis of a general recurrent neural network model for time-varying matrix inversion," IEEE Trans. Neural Netw., vol. 16, no. 6, pp. 1477-1490, 2005.

[12] Y. Zhang, "Revisit the analog computer and gradient-based neural system for matrix inversion," In Proceedings of IEEE international symposium on intelligent control, pp. 1411-1416, 2005.

[13] L. Xiao, Y. Zhang, K. Li, B. Liao, and Z. Tan, "A novel recurrent neural network and its finite-time solution to time-varying complex matrix inversion," Neurocomputing, vol. 331, pp. 483-492, 2019.

[14] Y. Zhang, K. Chen, and H. Tan, "Performance analysis of gradient neural network exploited for online time-varying matrix inversion," IEEE Trans. Autom. Contr., vol. 54, no. 8, pp. 1940-1945, 2009.

[15] L. Xiao, and Y. Zhang, "Solving time-varying inverse kinematics problem of wheeled mobile manipulators using Zhang neural network with exponential convergence," Nonlinear Dyn., vol. 76, no. 2, pp. 1543-1559, 2014.

[16] L. Jin, S. Li, B. Liao and Z. Zhang, "Zeroing neural networks: A survey," Neurocomputing., vol. 267, pp. 597-604, 2017.

[17] L. Xiao, "A new design formula exploited for accelerating Zhang neural network and its application to time-varying matrix inversion," Theor. Comput. Sci., vol. 647, pp. 50-58, 2016.

[18] L. Jin, Y. Zhang, and S. Li, "Integration-enhanced Zhang neural network for real-time-varying matrix inversion in the presence of various kinds of noises," IEEE Trans. Neural Netw. Learning Syst., vol. 27, no. 12, pp. 2615-2627, 2016.

[19] L. Jin, Y. Zhang, S. Li, and Y. Zhang, "Noise-tolerant ZNN models for solving time-varying zero-finding problems: A control-theoretic approach," IEEE Trans. Autom. Control., vol. 62, no. 2, pp. 992-997, 2017.

[20] L. Jin, S. Li, B. Hu, M. Liu, J. Yu, "A noise-suppressing neural algorithm for solving the time-varying system of linear equations: A control-based approach," IEEE Trans. Ind. Inform., vol. 15, no. 1, pp. 236-246, 2019.
[21] L. Xiao, Y. Zhang, et al., "A new noise-tolerant and predefined-time ZNN model for time-dependent matrix inversion," Neural Netw., vol. 117, pp. 124-134, 2019.

[22] Q. Xiang, B. Liao, L. Xiao and L. Jin, "A noise-tolerant Z-type neural network for time-dependent pseudoinverse matrices," Optik, vol. 165, pp. $16-28,2018$.

[23] L. Xiao, "Design and analysis of robust nonlinear neural dynamics for solving dynamic nonlinear equation within finite time," Nonlinear Dyn., vol. 96, no. 4, pp. 2437-2447, 2019.

[24] L. Jin, S. Li, et al. "Cooperative motion generation in a distributed network of redundant robot manipulators with noises," IEEE Trans. Syst. Man. Cybern. Syst., vol. 48, pp. 1715-1724, 2018.

[25] P.S. Stanimirović, V.N. Katsikis, and S. Li, "Integration enhanced and noise tolerant ZNN for computing various expressions involving outer inverses," Neurocomputing, vol. 329, pp. 129-143, 2019.

[26] L. Xiao, S. Li, J. Yang, and Z. Zhang, "A new recurrent neural network with noise-tolerance and finite-time convergence for dynamic quadratic minimization," Neurocomputing, vol. 285, pp. 125-132, 2018.

[27] L. Xiao, S. Li, F. Lin, Z. Tan, A.H. Khan, "Zeroing neural dynamics for control design: Comprehensive analysis on stability, robustness, and convergence speed," IEEE Trans. Ind. Inform., vol. 15, no. 5, pp. 26052616, 2019.

[28] L. Xiao, B. Liao, Q. Zeng, L. Ding, and R. Lu, "A complex gradient neural dynamics for fast complex matrix inversion," International Symposium on Neural Networks (Springer), pp. 521-528, 2017.

[29] L. Jin, S. Li, B. Hu, M. Liu, and J. Yu, "Noise-suppressing neural algorithm for solving time-varying system of linear equations: A controlbased approach," IEEE Trans. Ind. Inform., vol. 15, no. 1, pp. 236-246, Jan. 2019.

[30] S. Qiao, X. Wang, and Y. Wei, "Two finite-time convergent Zhang neural network models for time-varying complex matrix Drazin inverse," Linear Algebra Appl., vol. 542, pp. 101-117, 2018.

[31] L. Xiao, and R. Lu, "A fully complex-valued gradient neural network for rapidly computing complex-valued linear matrix equations," Chinese J. Electron., vol. 26, no. 6, pp. 1194-1197, 2017.

[32] Y. Zhang, and D. Guo, "Time-varying complex matrix inverse," Springer Berlin Heidelberg, pp. 163-172. 2015.

[33] X. Wang, Y. Wei, and P.S. Stanimirovic, "Complex neural network models for time-varying drazin inverse," Neurocomputing, vol. 28, no. 12, pp. 2790-2824, 2016.

[34] L. Xiao, "A finite-time convergent neural dynamics for online solution of time-varying linear complex matrix equation," Neurocomputing, vol. 167, pp. 254-259, 2015.

[35] Z. Zhang, Z. Yan, and T. Fu, "Varying-parameter RNN activated by finite-time functions for solving joint-drift problems of redundant robot manipulators," IEEE Trans. Ind. Inform., vol. 14, no. 12, pp. 5359-5367, 2018.

[36] L. Xiao, and Y. Zhang, "Zhang neural network versus gradient neural network for solving time-varying linear inequalities," IEEE Trans Neural Netw., vol. 22, no. 10, pp. 1676-1684, 2011.

[37] Y. Zhang, and Z. Li, "Zhang neural network for online solution of timevarying convex quadratic program subject to time-varying linear-equality constraints," Phys. Lett. A., vol. 373, no. 18-19, pp. 1639-1643, 2009.

[38] L. Xiao, and B. Liao, "A convergence-accelerated Zhang neural network and its solution application to Lyapunov equation," Neurocomputing, vol. 193, pp. 213-218, 2016.

[39] H. Lu, L. Jin, et al., "RNN for solving perturbed time-varying underdetermined linear system with double bound limits on residual errors and state variables," IEEE Trans. Ind. Inform., In press, 2019.

[40] Y. Zhang, Z. Li, and K. Li, "Complex-valued Zhang neural network for online complex-valued time-varying matrix inversion," Appl. Math. Comput., vol. 217, no. 24, pp. 10066-10073, 2011.

[41] Z. Tan, Y. Hu, L. Xiao, and K. Chen, "Robustness analysis and robotic application of combined function activated RNN for time-varying matrix pseudo inversion," IEEE Access, vol. 7, pp. 33434-33440, 2019.

[42] F. Yu, L. Liu, L. Xiao, K. Li, and S. Cai, "A robust and fixed-time zeroing neural dynamics for computing time-variant nonlinear equation using a novel nonlinear activation function," Neurocomputing, vol. 350, pp. 108-116, 2019.

[43] J. Lian, and J. Wang, "Passivity of switched recurrent neural networks with time-varying delays," IEEE Trans. Neural Netw. Learning Syst., vol. 26, no. 2, pp. 357-366, 2015.

[44] Z. Zhang and Y. Zhang, "Design and experimentation of accelerationlevel drift-free scheme aided by two recurrent neural networks," IET Control Theory Appl., vol. 7, no. 1, pp. 25-42, 2013.

[45] A.V. Oppenheim and A.S. Willsky, "Signals \& Systems," Englewood Cliffs, NJ, USA: Prentice-Hall., 1997. 